\title{
Increased expression of hypoxia-inducible factor $1 \alpha$ in type I and type II endometrial carcinomas
}

\author{
Vaishali Pansare ${ }^{1}$, Adnan R Munkarah ${ }^{2}$, Veronica Schimp ${ }^{2}$, M Haitham Arabi ${ }^{1}$, \\ Ghassan M Saed ${ }^{1}$, Robert T Morris ${ }^{2}$ and Rouba Ali-Fehmi ${ }^{1}$ \\ ${ }^{1}$ Department Pathology, Wayne State University and Karmanos Cancer Institute, Detroit, MI, USA and \\ ${ }^{2}$ Department of Obstetrics and Gynecology, Wayne State University and Karmanos Cancer Institute, \\ Detroit, MI, USA
}

\begin{abstract}
Hypoxia-inducible factor $1 \alpha$ (HIF-1 $\alpha)$ is a nuclear protein that is upregulated in many tumors and triggers biologic events intimately associated with aggressive tumor behavior. The aim of this study was to analyze the expression of HIF-1 $\alpha$, vascular endothelial growth factor (VEGF), Ki-67 and p53 in type I and type II endometrial adenocarcinoma. In total, 149 patients diagnosed with endometrial adenocarcinoma in our institute from 1995 to 2001 were included in this study, of which 108 were type I and 41 were type II endometrial adenocarcinoma. Patient demographics, clinical and pathological data were reviewed. Tissue microarrays were prepared from the paraffin blocks and immunohistochemistry was performed for antibodies against HIF-1 $\alpha$, VEGF, Ki-67 and p53. High expression of HIF-1 $\alpha$, VEGF, Ki-67 and p53 were significantly more frequent in type II than type I endometrial adenocarcinoma $(P<0.001)$. HIF-1 $\alpha$ expression was highly correlated with VEGF expression in the tumor cells $(P=0.001)$. In type I endometrial adenocarcinoma, high expression of HIF-1 $\alpha$ showed a significant correlation with higher grade of the tumor, depth of myometrial invasion, adnexal invasion and clinical stage. A similar correlation was not observed in type II endometrial adenocarcinoma. Surgical stage was the only independent prognostic marker for survival. In conclusion, high expression of HIF- $1 \alpha$ is more frequent in type II than in type I endometrial adenocarcinoma. In type I endometrial adenocarcinoma, HIF-1 $\alpha$ expression correlates with morphologic features of aggressiveness. In type II endometrial adenocarcinoma, there is no correlation between HIF- $1 \alpha$ expression and these features. Thus, HIF-1 $\alpha$ may play an important role in endometrial adenocarcinoma progression, particularly in type I endometrial adenocarcinoma. Additional investigations of HIF- $1 \alpha$ as a biomarker of aggressive potential and as a novel target for therapeutics in endometrial adenocarcinoma are warranted.

Modern Pathology (2007) 20, 35-43. doi:10.1038/modpathol.3800718; published online 10 November 2006
\end{abstract}

Keywords: high expression of HIF-1 1 ; endometrial carcinoma; type I; type II

Endometrial cancer is the most common invasive neoplasm of the female genital tract. Worldwide, approximately 150000 new cases are diagnosed each year, making endometrial carcinoma the fifth most common cancer in women. ${ }^{1}$ Based on the clinicopathologic, immunohistochemical and molecular genetic studies, endometrial carcinomas have been classified into two major types: type I and type II endometrial adenocarcinoma. ${ }^{1}$ Type I carcinomas have an endometrioid or mucinous histology, are

Correspondence: Dr R Ali-Fehmi, MD, Department of Pathology, Wayne State University and Karmanos Cancer Institute, 3990 John R, Detroit, MI 48201, USA

E-mail: rali@med.wayne.edu

Received 23 June 2006; revised 22 September 2006; accepted 26 September 2006; published online 10 November 2006 usually related to estrogen stimulation and may be associated with PTEN mutation. Type II carcinomas, on the other hand, are aggressive, not related to estrogen stimulation and are associated with $p 53$ mutation. Serous carcinomas and clear cell carcinomas are included in type II endometrial adenocarcinoma. ${ }^{1}$

Hypoxia-inducible factor 1 (HIF-1) is a nuclear protein that activates gene transcription specifically in response to reduced cellular oxygen concentration and therefore acts as a marker for hypoxia. It is a dimeric protein with two subunits, $\alpha$ and $\beta$. HIF- $1 \alpha$ is the specific hypoxia-regulated subunit. ${ }^{2}$ Hypoxia results in stabilization of HIF- $1 \alpha$, facilitating nuclear HIF- $1 \alpha$ binding and restoration of oxygen homeostasis by inducing glycolysis and angiogenesis through upregulation of vascular endothelial growth 
factor (VEGF). ${ }^{3}$ Experimental and clinical evidence suggest that the hypoxic fraction in solid tumors reduces sensitivity to conventional treatment modalities, influences growth and may increase malignant progression. ${ }^{3,4}$ Importantly, hypoxia has been clinically demonstrated to predict an adverse treatment outcome in the radiotherapeutic management of cancer of the head and neck, uterine cervix and soft tissue sarcomas. ${ }^{5-10}$ Upregulation of HIF- $1 \alpha$ has also been identified in low-stage endometrioid adenocarcinomas. ${ }^{11}$ However, its role in other types of endometrial carcinoma and its association with prognosis and survival have not been well documented in literature.

The p53 tumor suppressor gene is closely associated with the regulation of angiogenesis. ${ }^{12}$ Overexpression of p53 protein has been shown to be an independent poor prognostic indicator associated with 5-year survival in patients with endometrial cancer and an increased likelihood of distant failure. ${ }^{12-14}$

$\mathrm{Ki}-67$ is a nuclear antigen that is a marker of cellular proliferation. Antibodies directed against the DNA-binding nuclear protein, Ki-67 antigen, identify cells in most of $G_{1}$, and all of $S, G_{2}$ and $M$ phases of cell cycle, but not in $G_{0}$ phase. It has also been found to be a potential prognostic factor for survival in several human cancers, including endometrial cancer. ${ }^{15}$

The aim of this study was to (1) determine the expression of HIF1 $\alpha$, VEGF, p53 and Ki-67 in the two different types of endometrial carcinomas, namely type I and type II and (2) analyze the impact of high expression on histological features of tumor aggressiveness.

\section{Materials and methods}

Using the database from the Division of Gynecologic Oncology and the Department of Pathology, we identified 149 patients who were diagnosed with endometrial cancer and underwent primary surgery at our institution between 1995 and 2001. After obtaining approval from the Institutional Review Board, a retrospective chart review of patients' demographic, clinical and pathological data was performed. In cases missing survival data, the follow-up status was obtained through the SEER database.

Surgical staging was determined using the criteria recommended by the International Federation of
Gynecology and Obstetrics (FIGO). The histological type was determined using the World Health Organization (WHO) criteria. The tumors were classified into type I (endometrioid and mucinous) and type II (serous and clear cell) as described by Kurman. ${ }^{1}$ No mucinous or clear cell carcinomas were included in this study. Microscopic grading was based on FIGO grading system. A tumor was considered as FIGO grade 1 when no more than 5\% of the tumor was composed of solid masses; as FIGO grade 2 when $6-50 \%$ of tumor was composed of solid masses and as FIGO grade 3 when $>50 \%$ of tumor was composed of solid masses. Nuclear grade was determined by the variation in nuclear size and shape, chromatin distribution and size of nucleoli. ${ }^{1}$

Tissue microarrays (Manual Tissue Microarrayer, number 1, Beecher Instruments) were prepared using selected paraffin-embedded blocks of tumor from each case. A single block was selected per case and from each of these paraffin blocks, three $1.5 \mathrm{~mm}$ in diameter cores were obtained. Using standard immunohistochemistry (IHC) techniques, all the microarray blocks were stained with antibodies for HIF-1 $\alpha$ (H1- $\alpha 67$, Novus-Biologica), VEGF (C1, Santa Cruz), Ki-67 (MM1, Ventana) and p53 (DO7, Ventana) using standard techniques, as listed in Table 1.

\section{Assessment of Antibody Expressions}

Each section was deparaffinized and subjected to immunohistochemical staining, with standard streptavidin-biotin-peroxidase techniques, with diaminobenzidine as the chromogen. For VEGF: 4-5 $\mu$ thick sections were antigen retrieved by steam treatment in a citrate buffer, quenched for $10 \mathrm{~min}$ with $3 \%$ hydrogen peroxide, preincubated with blocking serum at $1: 20$ in $2 \%$ bovine serum albumin/phosphate-buffered saline solution for $15 \mathrm{~min}$ at room temperature. After incubation with the primary antibody VEGF, slides were rinsed with PBS, and the secondary antibody was applied at 1:500 in PBS for $30 \mathrm{~min}$ at room temperature. After rinses with PBS for $30 \mathrm{~s}$, slides were incubated with streptavidin/peroxidase at 1:500 in PBS for $30 \mathrm{~min}$ at room temperature, then rinsed with PBS and incubated for $15 \mathrm{~min}$ with $0.06 \%$ diaminobenzidine and counterstained with Harris-modified hematoxylin (Fisher Healthcare, Hanover Park, IL, USA). For p53, a dilution of 1:100 was used for the primary antibody. For Ki-67 and HIF-1 $\alpha, 4-5 \mu$ sections were

Table 1 Immunohistochemical methods

\begin{tabular}{|c|c|c|c|c|c|}
\hline & Antibody & Company & Antigen retrieval & Concentration & Primary incubation \\
\hline HIF1 $\alpha$ & H1-alpha67 & Novus-Biologicals & CC2, citrate & 1:2000 (ovary) & Benchmark 32 min \\
\hline VEGF & C-1 & Santa Cruz & Protease I, $10 \mathrm{~min}$ & $1: 50$ & Nexes, $32 \mathrm{~min}$ \\
\hline Ki-67 & $\mathrm{K}-2$ & Ventana & CC1, EDTA & Predilute & Benchmark 16 min \\
\hline P53 & DO7 & Ventana & CC1, EDTA & Predilute & Benchmark 32 min \\
\hline
\end{tabular}


antigen retrieved by using an EDTA buffer. The primary antibody for Ki-67 was used at a dilution of 1:100 and for HIF-1 $\alpha$ a dilution of 1:3000 was used.

Both the H\&E slides and the immunohistochemical stains were evaluated in a blinded fashion separately by two pathologists (RAF and VP). In cases where there was a discrepancy, the slides were reviewed together by both pathologists on a doubleheaded scope and a consensus diagnosis was recorded. For all the immunohistochemical markers, the percentage of cells staining was recorded. Tumors were considered positive if more than $10 \%$ of the cells evaluated expressed the antibody. Positive tumors were further categorized into groups by the percent of cells stained..$^{7-10}$

HIF-1 $\alpha$ was expressed in both nuclei and cytoplasm. Expression of HIF-1 $\alpha$ was assessed by analyzing at least 1000 tumor cells from tumor fields, and the labeling index was calculated as the percentage of labeled nuclei of the total number of tumor cells that were counted. HIF- $1 \alpha$ expression was considered low if $>10 \%$ but $<45 \%$ (median value) of cells showed positive nuclear or cytoplasmic staining, those with $>45 \%$ (median value) were considered to have high expression. ${ }^{16,17}$ For VEGF assessment, the staining intensity and the percentage of tumor cells that were stained were analyzed. Staining intensity was scored as 0 (negative), $1+$ (weak), $2+$ (medium) and $3+$ (strong). A combined score that was based on the staining intensity and the percentage of cells stained was used to assign a final score. Low expression was defined as intensity $0,1,2$ or 3 and $<10 \%$ cells staining or intensity 0 , 1 and $<50 \%$ cells staining; high expression was defined as intensity 2,3 and $>10 \%$ cells staining or intensity $1,2,3$ and $>50 \%$ of cells staining. ${ }^{18}$

To determine Ki-67 and p53 expression, nuclei from at least 1000 tumor cells were counted from the tumor fields, and the labeling index was calculated as the percentage of labeled nuclei of the total number of tumor cells that were counted. ${ }^{19}$ Tumors with a Ki-67 labeling index above 44\% (median value) were considered to have high proliferation indices. ${ }^{19}$

\section{Statistical Analysis}

Statistical analyses and graphs were performed using Statistical Software Package for the Social Sciences, for Windows version 13.0 (SPSS, Inc., Chicago, IL, USA). Spearman's correlation and Fisher's exact test were used to analyze the expression of IHC markers and to their relation to adnexal metastasis, vascular and/or myometrial invasion. Survival times were estimated in months from the date of diagnosis to the date of death or last followup. Survival curves were plotted using the KaplanMeier method, and the log-rank test and Cox Regression (forward stepwise) were used to determine statistical differences in survival between the various groups. Statistical significance was defined as a probability value less than 0.05 .

\section{Results}

The study cohort consisted of 149 patients with 108 type I and 41 type II endometrial adenocarcinomas. The mean age at diagnosis was 61 years (38-79 years) for type I patients and 67 years (56-83 years) for type II endometrial adenocarcinoma patients $(P=\mathrm{NS})$. The patient demographics and tumor characteristics are listed in Table 2. Median followup of all patients was 52 months (range, 1-102 months).

IHC analysis revealed a significant difference in high expression of HIF- $1 \alpha$ between type I and type II endometrial adenocarcinoma (26\% of type I revealed high expression compared to $86 \%$ of type II; $P<0.001$ ) (Table 3; Figures 1 and 2). Similarly, type II tumors were more likely to exhibit high VEGF expression than type I tumors (Table 3; Figures 2 and 3$)$.

Table 2 Patient and tumor characteristics

\begin{tabular}{|c|c|c|c|}
\hline & Tуре I & Tyре II & $\mathrm{P}$-value \\
\hline No. of patients & $108(72.5 \%)$ & $41(27.5 \%)$ & \\
\hline Age (mean \pm s.d.) & $61.0 \pm 11.0$ & $67.4 \pm 12.5$ & 0.00 \\
\hline \multicolumn{4}{|l|}{ Race } \\
\hline Caucasian & $76(70 \%)$ & $22(54 \%)$ & \multirow[t]{2}{*}{0.054} \\
\hline African-American & $32(30 \%)$ & $19(46 \%)$ & \\
\hline \multicolumn{4}{|l|}{ Stage ${ }^{\mathrm{a}}$} \\
\hline I & $84(78 \%)$ & $17(44 \%)$ & \multirow[t]{5}{*}{$<0.001$} \\
\hline II & $8(7 \%)$ & $5(13 \%)$ & \\
\hline III & $12(11 \%)$ & $14(33 \%)$ & \\
\hline IV & $1(1 \%)$ & $5(10 \%)$ & \\
\hline No data available & $3(3 \%)$ & 0 & \\
\hline \multicolumn{4}{|l|}{ Outcome } \\
\hline Alive & $83(77 \%)$ & $20(49 \%)$ & \multirow[t]{3}{*}{0.001} \\
\hline Dead & $24(22 \%)$ & $21(51 \%)$ & \\
\hline No data available & $1(1 \%)$ & 0 & \\
\hline \multicolumn{4}{|c|}{ Histologic grade (FIGO) } \\
\hline 1 & $42(39 \%)$ & \multirow[t]{3}{*}{$\mathrm{N} / \mathrm{A}$} & \\
\hline 2 & $47(43.5 \%)$ & & \\
\hline 3 & $19(17.5 \%)$ & & \\
\hline \multicolumn{4}{|l|}{ Depth of invasion } \\
\hline None & $31(29 \%)$ & $8(7 \%)$ & \multirow[t]{4}{*}{0.047} \\
\hline Inner & $42(39 \%)$ & $15(33 \%)$ & \\
\hline Outer & $28(26 \%)$ & $18(60 \%)$ & \\
\hline No data available & $7(6 \%)$ & 0 & \\
\hline \multicolumn{4}{|l|}{ Vascular invasion } \\
\hline Yes & $41(39 \%)$ & $25(61 \%)$ & \multirow[t]{3}{*}{0.015} \\
\hline No & $64(59 \%)$ & $15(37 \%)$ & \\
\hline No data available & $3(2 \%)$ & $1(2 \%)$ & \\
\hline \multicolumn{4}{|l|}{ Adnexal invasion } \\
\hline Yes & $9(8 \%)$ & $13(31 \%)$ & \multirow[t]{3}{*}{0.002} \\
\hline No & $96(89 \%)$ & $28(69 \%)$ & \\
\hline No data available & $3(3 \%)$ & 0 & \\
\hline
\end{tabular}

${ }^{\mathrm{a}}$ FIGO clinical stage. 
Spearman's correlation test showed a significant correlation between HIF-1 $\alpha$ and VEGF expression in tumor cells $(P<0.001)$ (Figure 4$)$.

In type I endometrial adenocarcinoma, high expression of HIF- $1 \alpha$ showed significant correlation with parameters of tumor aggressiveness including myometrial and vascular invasion and adnexal metastasis (Table 4; Figure 5). High expression was seen in $24 \%$ of high-grade tumors (FIGO grades 2 and 3 ), $34 \%$ of those with outer half of myometrial invasion, $89 \%$ of those with adnexal invasion and $77 \%$ of those with high-stage tumors $(P=0.009$, $0.05,0.02$ and $<0.01$, respectively). A total of $27 \%$ of tumors with vascular invasion and $14 \%$ of tumors without vascular invasion showed high expression of HIF- $1 \alpha(P=0.06)$. When the same parameters of tumor aggressiveness were analyzed in type II

Table 3 High and low expression of HIF-1 $\alpha$, VEGF, Ki-67 and p53 in type I and type II endometrial adenocarcinoma

\begin{tabular}{|c|c|c|c|}
\hline $\begin{array}{l}\text { Immunohistochemical } \\
\text { marker }\end{array}$ & $\begin{array}{c}\text { Type I } \\
(\mathrm{n}=108)\end{array}$ & $\begin{array}{l}\text { Type II } \\
(\mathrm{n}=41)\end{array}$ & $\mathrm{P}$-value \\
\hline \multicolumn{4}{|c|}{ HIF $1 \propto(n=121$, missing 28$)$} \\
\hline Low & $60(75 \%)$ & $7(17 \%)$ & \multirow[t]{3}{*}{$<0.001$} \\
\hline High & $20(25 \%)$ & $34(83 \%)$ & \\
\hline Missing & 28 & 0 & \\
\hline \multicolumn{4}{|c|}{ VEGF ( $n=130$, missing 19) } \\
\hline Low & $61(69 \%)$ & $16(39 \%)$ & \multirow[t]{3}{*}{0.001} \\
\hline High & $28(31 \%)$ & $25(61 \%)$ & \\
\hline Missing & 19 & 0 & \\
\hline \multicolumn{4}{|c|}{ Ki-67 (n=128, missing 21$)$} \\
\hline Low & $54(61 \%)$ & $10(26 \%)$ & \\
\hline High & $35(39 \%)$ & $29(74 \%)$ & \\
\hline Missing & 19 & 2 & \\
\hline \multicolumn{4}{|l|}{ P53 ( $n=149$, missing 0$)$} \\
\hline Low & $90(83 \%)$ & $7(18 \%)$ & \multirow[t]{2}{*}{$<0.001$} \\
\hline High & $18(17 \%)$ & $34(82 \%)$ & \\
\hline
\end{tabular}

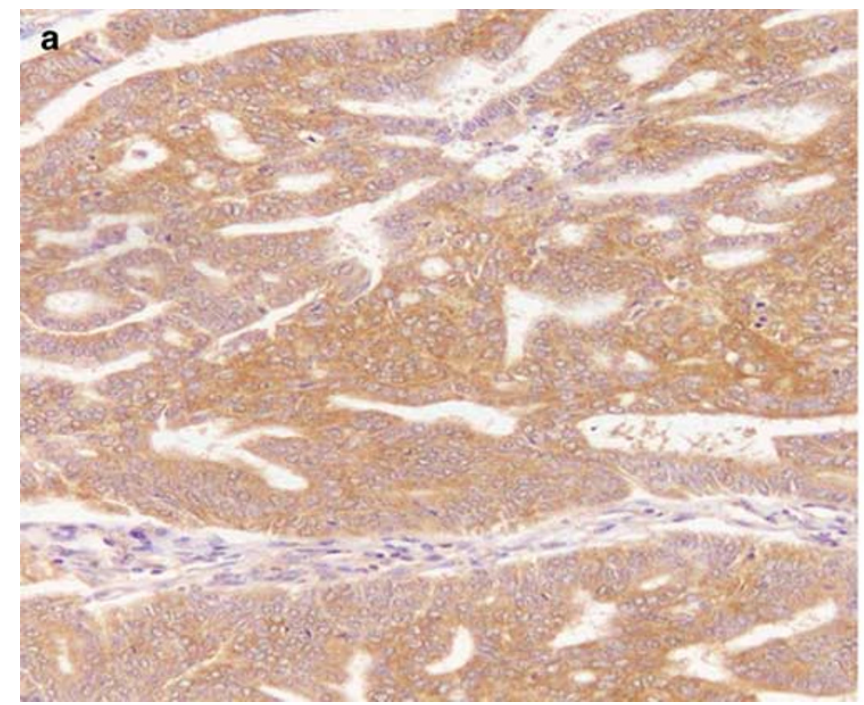

endometrial adenocarcinoma, high expression of HIF- $1 \alpha$ was observed in a significant number of cases, regardless of the myometrial invasion, adnexal invasion, vascular invasion and clinical stage $(P=$ NS) (Table 5; Figure 5).

Expression of VEGF followed a trend similar to HIF-1 $\alpha$. High expression of VEGF was significantly more in type II endometrial adenocarcinoma compared to type I endometrial adenocarcinoma (65 vs $32 \% ; P<0.001)$. Low expression of VEGF was noted in $68 \%(73 / 108)$ of type I endometrial adenocarcinoma and $35 \%(14 / 41)$ of type II endometrial adenocarcinoma $(P=0.001)$ (Table 3; Figures 2 and 3).

Expression of Ki-67 was statistically different in the two types of endometrial adenocarcinoma. High expression of Ki-67 was significantly different in type I and type II endometrial adenocarcinoma (37 vs $71 \% ; P<0.001$ ). Low expression of Ki-67 was observed in $63 \%(68 / 108)$ of type I and $29 \%$ of type II endometrial adenocarcinoma $(P<0.001)$ (Table 3; Figures 2 and 6).

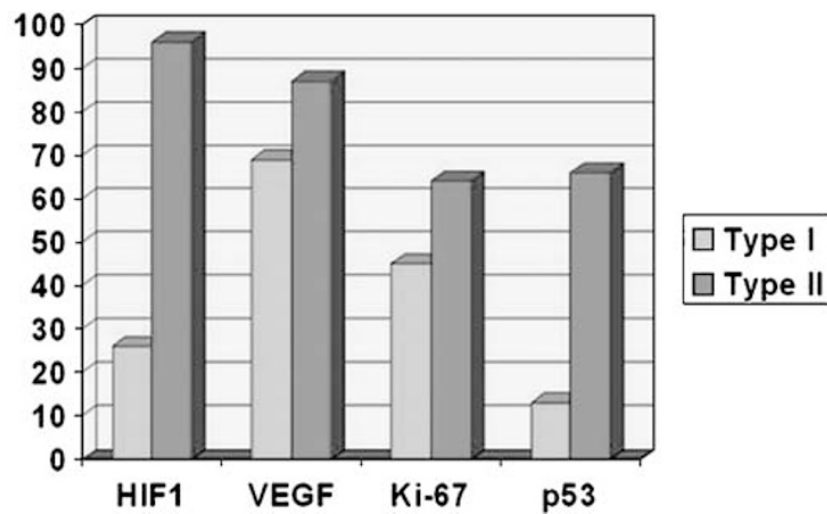

Figure 2 High expression of HIF-1 $\alpha$, VEGF, Ki-67 and p53 in type I (endometrioid, grade I, II and III) vs type II (serous) endometrial adenocarcinoma.

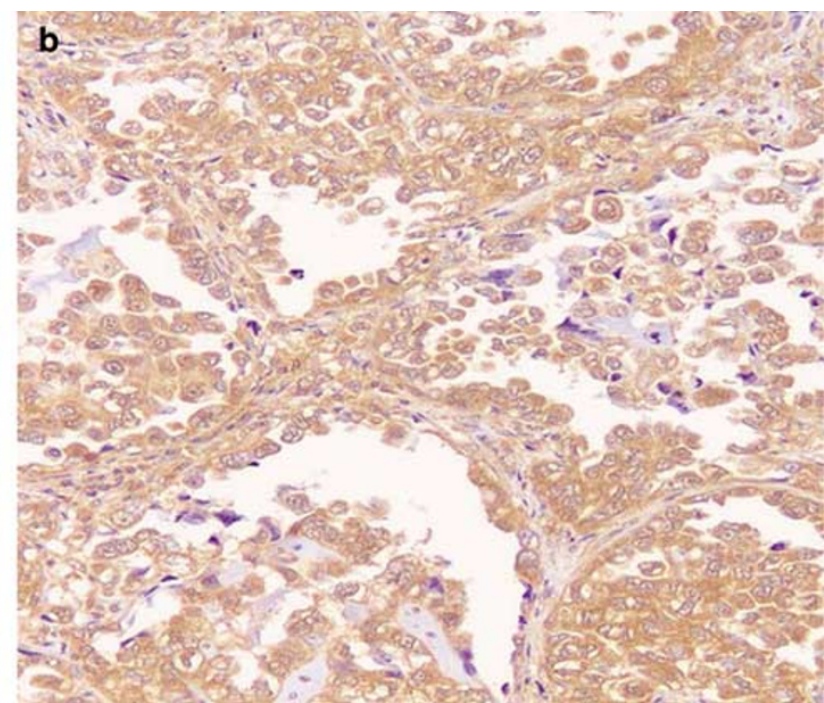

Figure 1 High expression of HIF- $1 \alpha$ in type I endometrial adenocarcinoma $(\mathbf{a} ; \times 400)$ and type II endometrial adenocarcinoma (b; $\times 400)$-composite picture. 

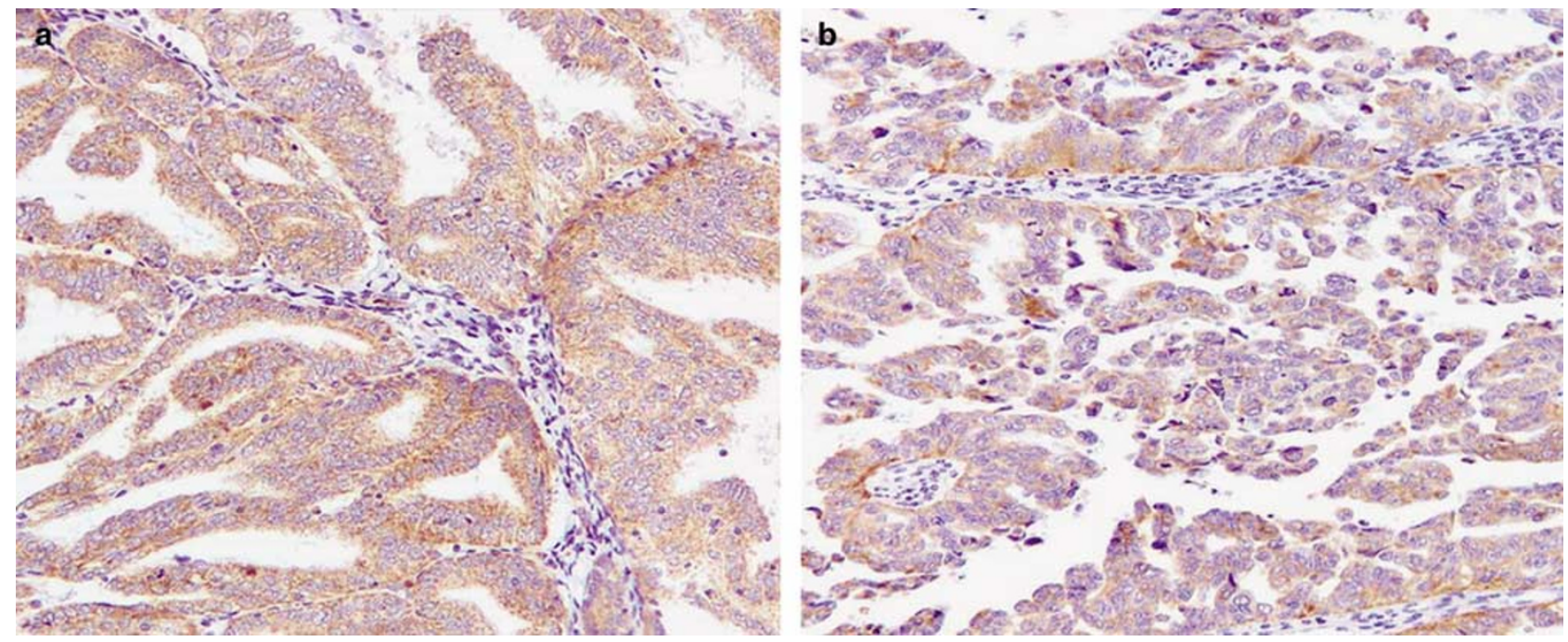

Figure 3 High expression of VEGF in type I endometrial adenocarcinoma (a) and type II endometrial adenocarcinoma (b)—composite picture.

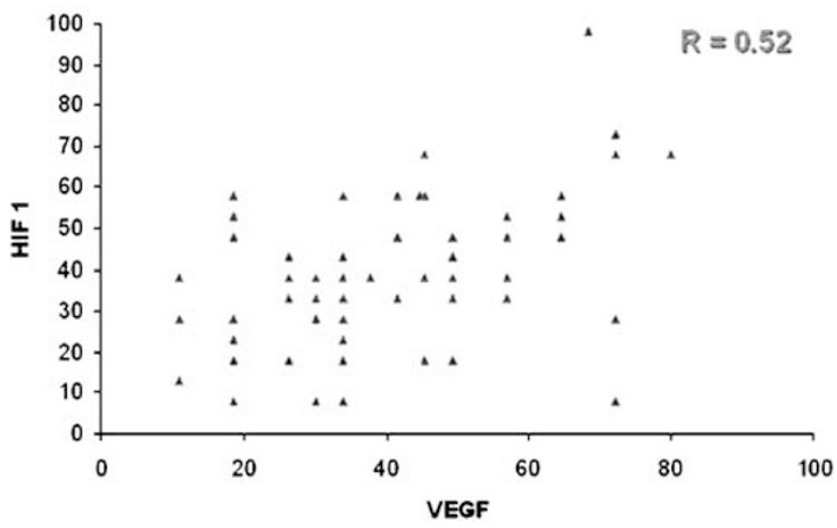

Figure 4 HIF-1 $\alpha$ expression was highly correlated with VEGF expression一 graph.

A significant difference was also noted in high expression of p53 in type I and type II endometrial adenocarcinoma (17 and $82 \% ; P<0.001)$. Low expression of p53 was noted in $83 \%(90 / 108)$ of type I endometrial adenocarcinoma, and only $18 \%$ $(7 / 41)$ of type II endometrial adenocarcinoma $(P<0.001)$ (Table 3; Figures 2 and 7).

On analyzing the expression of these four immunohistochemical markers with each other, we found a significant correlation of HIF- $1 \alpha$ with VEGF expression in tumor cells $(P<0.001)$ (Pearson correlation) (Figure 4).

The overall median survival of all patients $(n=149)$ was 53.4 months (range $=0.13-102$ months). The median survival of type I patients was 80 months compared to 53 months for those with type II endometrial adenocarcinoma $(P<0.001)$ (Figure 8). On univariate analysis, VEGF and HIF-1 $\alpha$ did not show correlation with survival $(P=\mathrm{NS}$; $t$ test). Using Cox regression analysis and including race, stage, grade and the molecular profile, only the
Table 4 High expression of HIF- $1 \alpha$ in type I endometrial adenocarcinoma and its correlation with tumor characteristics

\begin{tabular}{|c|c|c|}
\hline $\begin{array}{l}\text { Type I endometrial } \\
\text { adenocarcinoma }(\mathrm{n}=108)\end{array}$ & $\begin{array}{l}\text { High expression } \\
\text { of } H I F-1 \alpha \\
(\mathrm{n}=20)\end{array}$ & $\mathrm{P}$-value \\
\hline \multicolumn{3}{|l|}{ Grade ( $n=94$, missing 14$)$} \\
\hline Low $(n=42)$ & $4(9.5 \%)$ & \multirow[t]{2}{*}{0.009} \\
\hline $\operatorname{High}^{\mathrm{a}}(n=66)$ & $16(24 \%)$ & \\
\hline \multicolumn{3}{|c|}{ Myometrial invasion ( $n=101$, missing 7$)$} \\
\hline None $(n=31)$ & $5(16 \%)$ & \multirow[t]{3}{*}{0.05} \\
\hline Inner $(n=41)$ & $5(12 \%)$ & \\
\hline Outer $(n=29)$ & $10(34 \%)$ & \\
\hline \multicolumn{3}{|c|}{ Adnexal invasion $(n=105$, missing 3$)$} \\
\hline No $(n=96)$ & $12(12.5 \%)$ & \multirow[t]{2}{*}{0.02} \\
\hline Yes $(n=9)$ & $8(89 \%)$ & \\
\hline \multicolumn{3}{|c|}{ Vascular invasion $(n=105$, missing 3$)$} \\
\hline No $(n=64)$ & $9(14 \%)$ & \multirow[t]{2}{*}{0.06} \\
\hline Yes $(n=41)$ & $11(27 \%)$ & \\
\hline \multicolumn{3}{|l|}{ Stage $(n=105$, missing 3$)$} \\
\hline Low $(n=92)$ & $10(11 \%)$ & \multirow[t]{2}{*}{$<0.01$} \\
\hline High $(n=13)$ & $10(77 \%)$ & \\
\hline
\end{tabular}

${ }^{\mathrm{a}}$ High grade $=$ FIGO grades 2 and 3.

stage of disease remained a significant independent poor prognostic variable.

\section{Discussion}

HIF- $1 \alpha$ is a marker of hypoxia, which activates gene transcription in hypoxic states and stimulates angiogenesis in tumors. ${ }^{2,3,11,20}$ It has been shown to be overexpressed in tumors from various anatomic locations such as cervical squamous cell carcinomas, head and neck cancers and soft tissue tumors, 

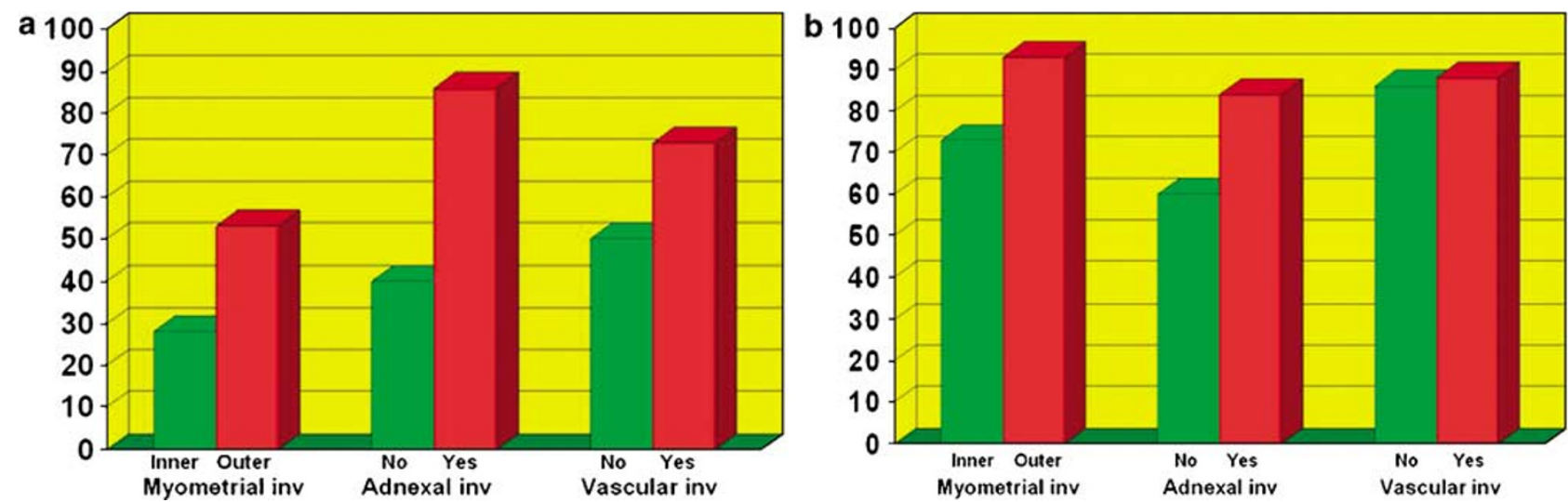

Figure 5 High expression of HIF-1 $\alpha$ correlated with tumor aggressive properties in type I endometrial adenocarcinoma (a) and type II endometrial adenocarcinoma (b)—composite picture.

Table 5 High expression of HIF- $1 \alpha$ in type II endometrial adenocarcinoma and its correlation with tumor characteristics (Spearman's correlation and Fisher's exact test)

\begin{tabular}{|c|c|c|}
\hline $\begin{array}{l}\text { Type II endometrial } \\
\text { adenocarcinoma }(\mathrm{n}=41)\end{array}$ & $\begin{array}{l}\text { High expression } \\
\text { of } H I F-1 \alpha(\%)\end{array}$ & $\mathrm{P}$-value \\
\hline \multicolumn{3}{|l|}{ Grade } \\
\hline High & - & - \\
\hline \multicolumn{3}{|l|}{ Myometrial invasion } \\
\hline None $(n=13)$ & $10(77 \%)$ & \\
\hline Inner $(n=10)$ & $7(70 \%)$ & NS \\
\hline Outer $(n=18)$ & $17(94 \%)$ & \\
\hline \multicolumn{3}{|c|}{ Adnexal Invasion ( $n=39$, missing 2$)$} \\
\hline No $(n=27)$ & $24(89 \%)$ & NS \\
\hline Yes $(n=12)$ & $10(83 \%)$ & \\
\hline \multicolumn{3}{|c|}{ Vascular invasion ( $n=40$, missing 1$)$} \\
\hline No $(n=15)$ & $12(80 \%)$ & NS \\
\hline Yes $(n=25)$ & $22(88 \%)$ & \\
\hline \multicolumn{3}{|l|}{ Stage $(n=39$, missing 2$)$} \\
\hline Low $(n=22)$ & $19(86 \%)$ & NS \\
\hline High $(n=17)$ & $15(88 \%)$ & \\
\hline
\end{tabular}

colon cancers, etc. ${ }^{5,6,8,9,14}$ Tumor hypoxia through activation of angiogenesis helps in tumor progression and metastasis. It makes solid tumors resistant to radiation and chemotherapy., ${ }^{4,5}$, This has been well studied in the head and neck, and uterine cervix. $^{5-7}$ Significant correlation of HIF- $1 \alpha$ was also noted with radiotherapy treatment of oropharyngeal and cervical carcinomas. ${ }^{5-8,21}$ Overexpression of HIF- $1 \alpha$ has also been described in endometrial carcinomas. ${ }^{11}$ Sivridis et al found HIF- $1 \alpha$ overexpression in $49 \%$ of 81 low-stage endometrioid adenocarcinomas (type I endometrial adenocarcinoma). Type II endometrial adenocarcinoma were not included in this study and hence comparative data for type I and type II endometrial adenocarcinoma were not analyzed. The findings of our study support those of the Sivridis study. High expression of HIF- $1 \alpha$ was noted in over $80 \%$ of type II endometrial adenocarcinoma and only $26 \%$ of type I cancers. To the best of our knowledge, this is the first study comparing the expression of HIF- $1 \alpha$ in type I and type II endometrial adenocarcinomas. The overexpression of HIF- $1 \alpha$ in type II endometrial adenocarcinoma supports the finding that HIF- $1 \alpha$ is associated with higher grade and more aggressive tumor histology. Acs et al studied HIF-1 $\alpha$ expression in endometrial cancers, including endometrioid, serous and clear cell carcinomas and found $74 \%$ of their tumors to be positive for the marker. This study did not compare type I vs type II endometrial adenocarcinoma. $^{22}$

Under hypoxic conditions, HIF- $1 \alpha$ binds to DNA at hypoxia response elements (HREs), thereby activating the vascular endothelial growth factor (VEGF) gene. ${ }^{23}$ Thus, HIF- $1 \alpha$ plays an important role in tumor angiogenesis, thus promoting tumor cell proliferation and growth and also helps in metastases. ${ }^{2} \mathrm{HIF}-1 \alpha$ has been shown to be the driving force in upregulating angiogenic pathways in non-smallcell lung carcinoma and nasopharyngeal carcinoma. ${ }^{20,24}$ In non-small-cell lung carcinoma, a strong association has been reported between HIF-1 $\alpha$ accumulation in cancer cells, tumor microvessel density and the expression of multiple angiogenic factors, including VEGF. ${ }^{16}$ Similarly Sivridis et $a l^{11}$ have shown an association between HIF- $1 \alpha$ overexpression and upregulation of VEGF in a cohort of stage I endometrioid-type endometrial adenocarcinoma. Our findings further support this intimate relationship between hypoxia and angiogenesis. A strong correlation was identified between HIF- $1 \alpha$ and VEGF expression in both type I and type II tumors. In the current study, VEGF expression was significantly higher in type II compared to type I endometrial adenocarcinoma. In a recent study on 70 stage 1 endometrioid adenocarcinomas, phosphorylated KDR expression was directly related to the HIF-1 $\alpha /$ VEGF pathway and to unfavorable prognosis. ${ }^{25}$

In the present study, high expression of Ki-67 was significantly different in the type I compared to type 

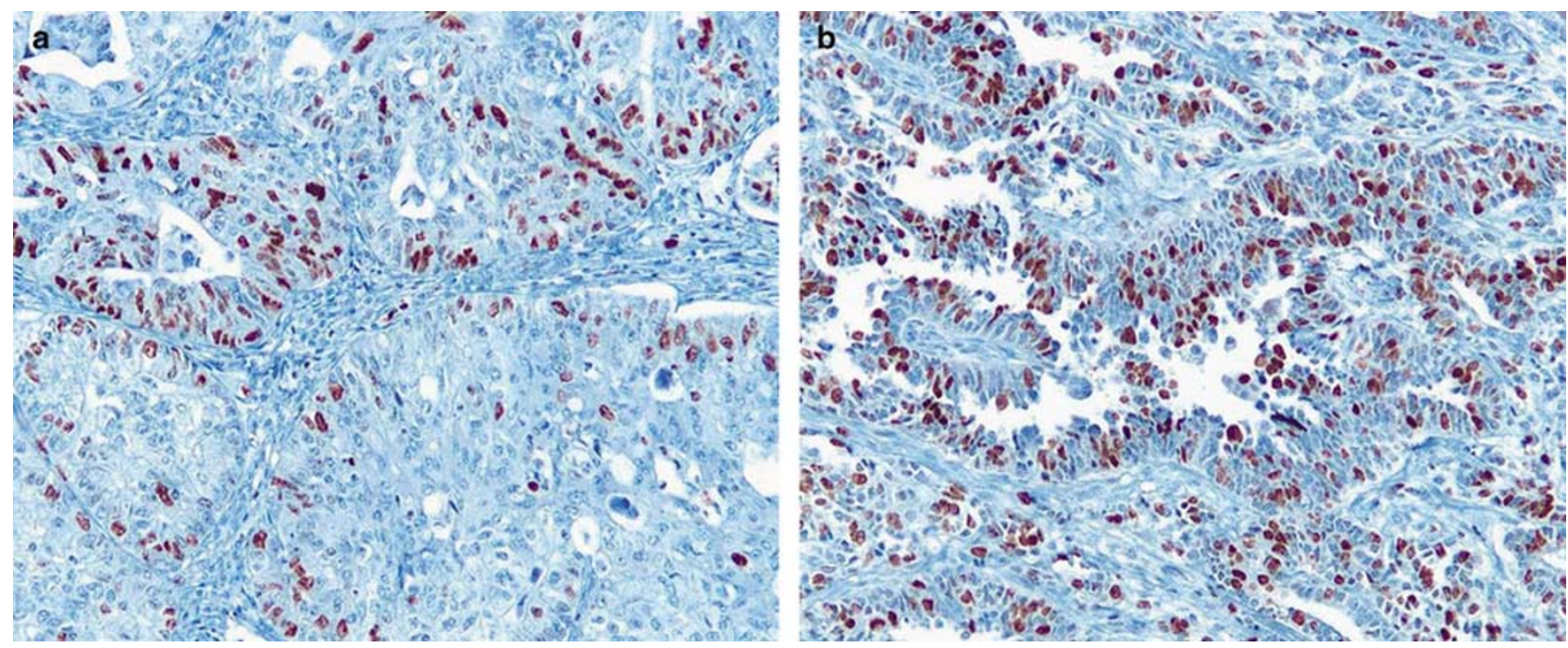

Figure 6 High expression of Ki-67 in type I endometrial adenocarcinoma (a) and type II endometrial adenocarcinoma (b)—composite picture.
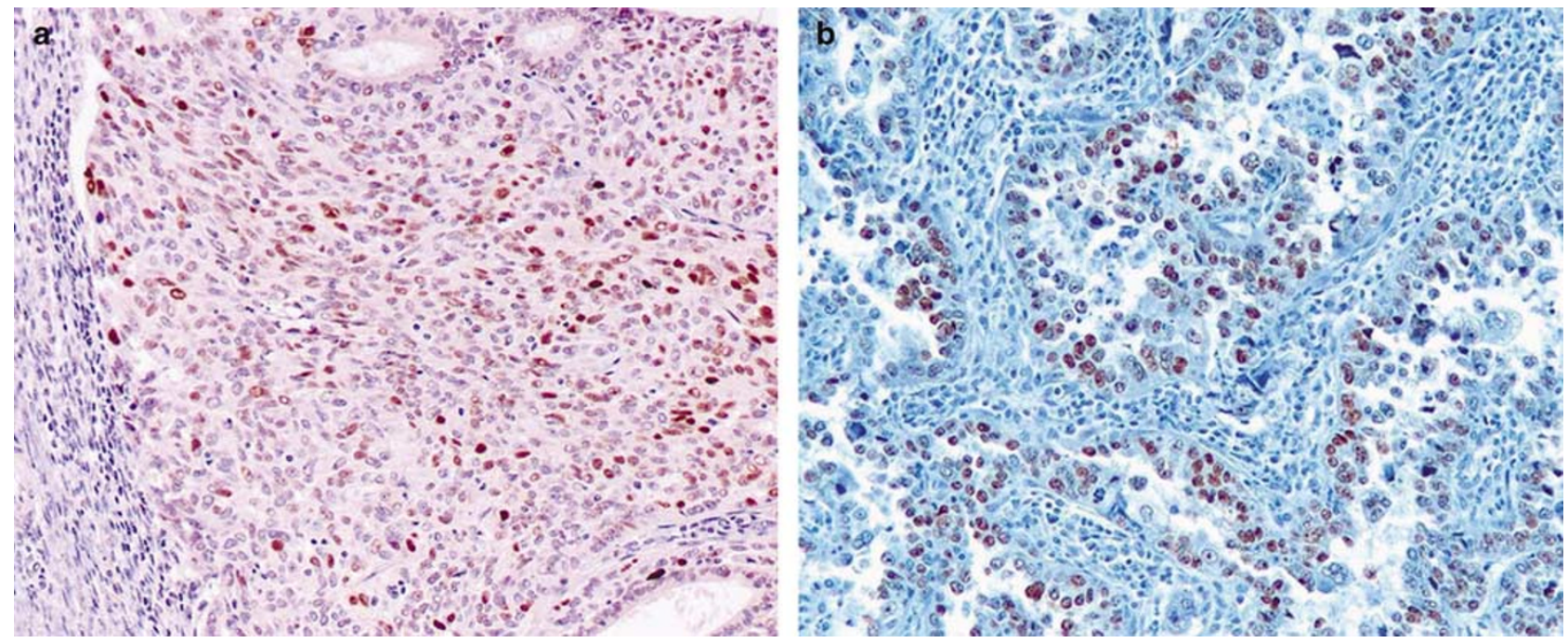

Figure 7 High expression of p53 in type I endometrial adenocarcinoma (a) and type II endometrial adenocarcinoma (b)—composite picture.

II endometrial adenocarcinoma, a tumor proliferation marker then correlates with tumor aggressiveness between the two types. In a prospective study of 115 patients treated for endometrial carcinoma, Salvesen et al showed a significant association of Ki-67 expression with FIGO stage, histologic type and histologic grade, and probability of survival by univariate analysis. In addition, using multivariate analysis, only Ki-67 expression and stage remained as significant independent prognosticators. ${ }^{26}$ Geisler et $a I^{27}$ found $\mathrm{Ki}-67$ to be an independent prognostic indicator predictive of survival by multivariate analysis in 147 patients with endometrial carcinoma. However in this study, correlation of Ki-67 with histologic type (endometrioid vs non-endometrioid), FIGO grade or clinical stage of the disease was not identified.
In the present study, an association between Ki-67 and HIF- $1 \alpha$ was not identified in type I or type II endometrial adenocarcinoma. Statistical correlation between HIF-1 $\alpha$ and Ki-67 expression was found in cancers of various organ sites including colon, breast, etc. by Zhong et al. ${ }^{16}$ This study did not include endometrial cancers. Another reason for noncorrelation could be the inherent high-grade nature of type II endometrial adenocarcinoma.

Mutations in the $p 53$ tumor suppressor gene occur in approximately $20-50 \%$ of type I endometrial cancers and up to $94 \%$ of type II endometrial cancers. ${ }^{12,13,28}$ Overexpression of p53 protein has been generally related to higher stage, aggressive cell types, increased histologic grade and depth of myometrial invasion. The overexpression of p53 in endometrial carcinomas demonstrated in the current 
Type I -Vs- Type II, Total Population

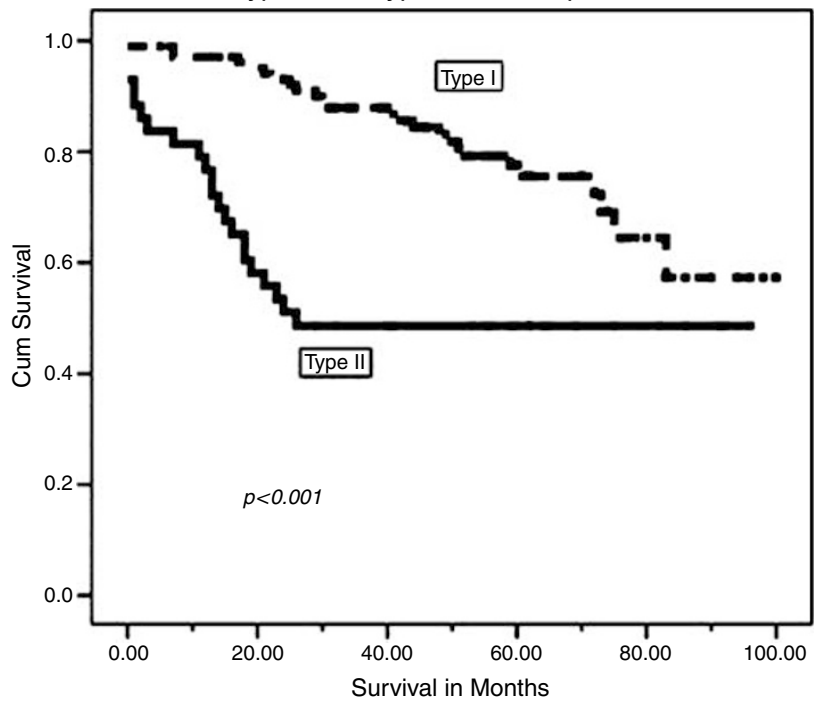

Figure 8 Survival of type I vs type II patients, total population (Kaplan-Meier method).

study further validates findings from the published literature. ${ }^{1,12,13,28}$ The current study also showed a significant difference in p53 expression between types I and II endometrial adenocarcinoma, with type II having greater frequency of high expression of the protein product. Few studies have analyzed the association of p53 with HIF-1 $\alpha$. In the present study, p53 expression was not associated with HIF- $1 \alpha$ expression in type I or type II endometrial adenocarcinoma. This is consistent with another study on 81 endometrioid-type endometrial adenocarcinomas, where HIF- $1 \alpha$ and p53 also failed to show any significant association. ${ }^{11}$ In contrast, Zhong et $a l^{16}$ found a correlation of HIF- $1 \alpha$ with p53 in common human cancers from various organ sites, although endometrial carcinoma was not included.

Studies evaluating HIF- $1 \alpha$ in other cancers have revealed that patients with tumors that express high levels of HIF- $1 \alpha$ have a poor outcome..$^{4-11,20}$ The negative impact of HIF- $1 \alpha$ protein overexpression on prognosis has been shown in cancers of head and neck, cervix and soft tissue sarcomas. ${ }^{5-10}$ It has been suggested that tumor hypoxia makes solid tumors resistant to radiation and chemotherapy. High expression of HIF-1 $\alpha$ was also associated with resistance to radiotherapy treatment of oropharyngeal and cervical carcinomas. ${ }^{4-7,21}$ In the current study, we found expression of HIF- $1 \alpha$ protein was correlated with aggressive tumor characteristics (higher grade of tumor, depth of tumor invasion and adnexal invasion) in type I endometrial adenocarcinoma, but failed to show similar correlation in type II endometrial adenocarcinoma. This may be due to the different biologic nature of these two types and the inherent high-grade property of type II endometrial adenocarcinoma. In our study popula- tion, only tumor stage was an independent prognostic marker. Birner et $a l^{17}$ found that HIF-1 $\alpha$ protein overexpression alone has no impact on the prognosis of ovarian cancer, but the combination of $H I F-1 \alpha$ with nonfunctional $p 53$ indicates a dismal prognosis in these tumors.

\section{Conclusion}

High expression of HIF- $1 \alpha$ is more frequent in type II than in type I endometrial adenocarcinoma.

In type I endometrial adenocarcinoma, HIF- $1 \alpha$ expression correlates with known pathologic features of tumor aggressiveness.

Thus, HIF- $1 \alpha$ may play an important role in endometrial cancer progression. Additional investigations of HIF- $1 \alpha$ as a biomarker of aggressive potential and as a novel target for therapeutics are warranted.

\section{References}

1 Kurman RJ (ed). Blaustein's Pathology of the Female Genital Tract, 5th edn. Springer: New York, 2002, pp 501-560.

2 Wang GL, Jiang BH, Rue EA, et al. Hypoxia-inducible factor 1 is a basic helix-loop-helix-PAS heterodimer regulated by cellular oxygen tension. Proc Natl Acad Sci USA 1995;92:5510-5514.

3 Carmeliet P, Dor Y, Herbert JM, et al. Role of HIF-1 alpha in hypoxia-mediated apoptosis, cell proliferation and tumor angiogenesis. Nature 1998;394: 485-490.

4 Vaupel P, Kelleher DK, Hockel M. Oxygen status of malignant tumors: pathogenesis of hypoxia and significance for tumor therapy. Semin Oncol 2001;28: 29-35.

5 Hockel M, Schlenger K, Aral B, et al. Association between tumor hypoxia and malignant progression in advanced cancer of the uterine cervix. Cancer Res 1996;56:4509-4515.

6 Brizel DM, Sibley GS, Prosnitz LR, et al. Tumor hypoxia adversely affects the prognosis of carcinoma of the head and neck. Int J Radiat Oncol Biol Phys 1997;38:285-289.

7 Brizel DM, Dodge RK, Clough RW, et al. Oxygenation of head and neck cancer: changes during radiotherapy and impact on treatment outcome. Radiother Oncol 1999;53:113-117.

8 Fyles AW, Milosevic M, Wong R, et al. Oxygenation predicts radiation response and survival in patients with cervix cancer. Radiother Oncol 1998;48:149-156.

9 Brizel DM, Scully SP, Harrelson JM, et al. Tumor oxygenation predicts for the likelihood of distant metastases in human soft tissue sarcoma. Cancer Res 1996;56:941-943.

10 Nordsmark M, Alsner J, Keller J, et al. Hypoxia in human soft tissue sarcomas: adverse impact on survival and no association with p53 mutations. Br J Cancer 2001;84:1070-1075.

11 Sivridis B, Giatromanolaki A, Gatter K, et al. Association of hypoxia-inducible factors $1 \alpha$ and $2 \alpha$ with activated angiogenic pathways and prognosis in 
patients with endometrial carcinoma. Cancer 2002;95:1055-1063.

12 Ravi R, Mookerjee B, Bhujwalla ZM, et al. Regulation of tumor angiogenesis by p53-induced degradation of hypoxia-inducible factor-1 alpha. Genes Dev 2000; 14:34-44.

13 Mariani A, Sebo TJ, Webb MJ, et al. Molecular and histopathologic predictors of distant failure in endometrial cancer. Cancer Detect and Prev 2003;27:434-441.

14 Geisler JP, Geisler HE, Wiemann MC, et al. p53 expression as a prognostic indicator of 5-year survival in endometrial cancer. Gynecol Oncol 1999;74:468-471.

15 Oreskovic S, Babic D, Kalafatic D, et al. A significance of immunohistochemical determination of steroid receptors, cell proliferation factor Ki-67 and protein p53 in endometrial carcinoma. Gynecol Oncol 2004; 93:34-40.

16 Zhong H, De Marzo A, Laughner E, et al. Overexpression of hypoxia-inducible factor $1 \alpha$ in common human cancers and their metastases. Cancer Res 1999;59:5830-5835.

17 Birner P, Schindl M, Obermair A, et al. Expression of hypoxia-inducible factor $1 \alpha$ in epithelial ovarian tumors: its impact on prognosis and on response to chemotherapy. Clin Cancer Res 2001;7:1661-1668.

18 Rouba Ali-Fehmi MC, Khalifeh I, Malone JM, et al. The effect of cyclooxygenase-2 expression on tumor vascularity in advanced stage ovarian serous carcinoma. Cancer 2003;98:1423-1429.

19 Mariani A, Sebo TJ, Katzmann JA, et al. Pretreatment assessment of prognostic indicators in endometrial cancer. Am J Obstet Gynecol 2000;182:1535-1544.

20 Giatromanolaki A, Koukourakis MI, Sivridis E, et al. Relation of hypoxia inducible factor 1alpha and 2 alpha in operable non-small cell lung cancer to angiogenic/molecular profile of tumors and survival. Br J Cancer 2001;85:881-890.

21 Burri P, Djonov V, Aebersold DM, et al. Significant correlation of hypoxia-inducible factor-1alpha with treatment outcome in cervical cancer treated with radical radiotherapy. Int J Gynecol Oncol Biol Phys 2003;56:494-501.

22 Acs G, Xu X, Chu C, et al. Prognostic significance of erythropoietin expression in human endometrial carcinoma. Cancer. 2004;100:2376-2386.

23 Semenza GL, Nejfelt MK, Chi SM, et al. Hypoxiainducible nuclear factors bind to an enhancer element located $3^{\prime}$ to the human erythropoietin gene. Proc Natl Acad Sci USA 1998;95:7987-7992.

24 Hui EP, Chan A, Pezella F, et al. Coexpression of hypoxia-inducible factors $1 \alpha$ and $2 \alpha$, carbonic anhydarse IX, and vascular endothelial growth factor in nasopharyngeal carcinoma and relationship to survival. Clin Cancer Res 2002;8:2595-2604.

25 Giatromanolaki A, Koukourakis MI, Turley H, et al, Tumor and Angiogenesis Research Group. Phosphorylated KDR expression in endometrial cancer cells relates to HIF1alpha/VEGF pathway and unfavourable prognosis. Mod Pathol 2006;19:701-707.

26 Salvesen HB, Iversen OE, Akslen LA. Identification of high-risk patients by assessment of nuclear Ki-67 expression in a prospective study of endometrial carcinomas. Clin Cancer Res 1998;4:2779-2785.

27 Geisler JP, Geisler HE, Miller GA, et al. MIB-1 in endometrial carcinoma: prognostic significance with 5-year follow-up. Gynecol Oncol 1999;75:432-436.

28 Tashiro $\mathrm{H}$, Isaacson $\mathrm{C}$, Levine $\mathrm{R}$, et al. p53 gene mutations are common in uterine serous carcinoma and occur in their pathogenesis. Am J Pathol 1997; 150:177-185. 